\title{
The indispensability argument and the nature of mathematical objects*
}

\author{
Matteo Plebani
}

Received: 31/01/2017

Final version: $28 / 03 / 2018$

BIBLID 0495-4548(2018)33:2p.249-263

DOI: $10.1387 /$ theoria. 17613

ABSTRACT: Two conceptions of the nature of mathematical objects are contrasted: the conception of mathematical objects as preconceived objects (Yablo 2010), and heavy duty platonism (Knowles 2015). It is argued that some theses defended by friends of the indispensability argument are in harmony with heavy duty platonism and in tension with the conception of mathematical objects as preconceived objects.

Keywords: indispensability argument; heavy duty platonism; metaphysical grounding.

RESUMEN: Se contrastan dos concepciones de la naturaleza de los objetos matemáticos: la concepción de los objetos matemáticos como objetos preconcebidos (Yablo 2010), y el platonismo de deber fuerte (Knowles 2015). Se argumenta que algunas de las tesis defendidas por los amigos del argumento de la indispensabilidad están en armonía con el platonismo de deber fuerte y en tensión con la concepción de los objetos matemáticos como objetos preconcebidos.

Palabras clave: argumento de la indispensabilidad; platonismo de deber fuerte; fundamentación metafísica.

\section{Introduction}

In recent philosophy of mathematics there has been a lively debate between proponents and critics of the so-called indispensability argument for the existence of mathematical objects (see Bangu 2017 and Colyvan 2015 for an overview).

The debate seems hard to settle. In an influential paper (Baker 2003), Alan Baker has put forward a hypothesis about why it is so difficult to make progress in the debate over the indispensability argument: "It may be that at the end of the day the dispute [...] stems not from any explicit thesis of platonism but from a certain background picture" (Baker 2003, 263).

This paper explores the tenability of (something close to) Baker's conjecture. I will contrast two conceptions of the nature of mathematical objects: the conception of mathematical objects as preconceived objects (Yablo 2010, Introduction), also called 'the preconceived view', and heavy duty platonism (Knowles 2015). I will then turn to the debate

* Many thanks to audiences in Santiago de Compostela, Munich and Pavia. This work has been financed by the Spanish Ministry of Economy and Competitiveness and FEDER through the projects FFI2013-41415-P (The explanatory function of abstract objects: their nature and cognoscibility), FFI2017-82534-P (Abstract Objects: For and Against. A Neo-Carnapian Way Out) and through a Juan de la Cierva-formación fellowship (IJCI-2014-19943). 
between supporters and critics of the indispensability argument. I will argue that there are some theses friends of the indispensability argument need to subscribe to, in order to respond to some criticisms raised against the indispensability argument and that such theses are in harmony with heavy duty platonism and in tension with the preconceived view. This should provide some evidence in favor of the conjecture that friends and critics of the indispensability argument might be attracted by different metaphysical views about the nature of mathematical objects.

Sections 1 and 2 introduce and discuss two conceptions of the nature of mathematical objects: the conception of mathematical objects as preconceived objects (section 1) and heavy duty platonism (section 2). According to the preconceived view (Yablo 2010, Introduction) mathematical objects have their properties fixed by what Yablo calls their 'job description': the way mathematical objects are characterized. Heavy duty platonism (HDP), on the other hand, is the view that "physical magnitudes, such as mass and temperature, are cases of physical objects being related to numbers" (Knowles 2015, 1255) and that such relations are metaphysically fundamental, i.e. not "derivative of [...] properties or relations that hold of physical objects alone" (Knowles 2015, 1255). Section 3 stresses that one important difference between the two views has to do with the question of whether the relations between abstract and concrete objects are grounded in purely concrete properties of the concrete objects or not. Section 4 presents a minimal version of the indispensability argument (IA) and connects it to the question whether the existence of mathematical objects makes a difference to the concrete, physical world (Baker 2003). In section 5 I argue that 'make no difference' claims (MND) should be understood in terms of the notions of orthogonality and subject matter, which I will define following Lewis (1988) and Yablo $(2012,2014)$. I will argue that the real issue that divides friends and foes of IA is whether the issue of how the concrete world is and the issue of whether there are mathematical objects are orthogonal or not. I will argue that friends of the indispensability argument should claim that the two issues are not orthogonal, as maintained by heavy duty platonism. On the other hand, to reject orthogonality and adopt the preconceived view yields an unstable position. Section 6 is dedicated to another theme closely related with the debate over IA: the question whether there are mathematical explanations of empirical phenomena. I will make the obvious observation that there are many ways mathematics can be explanatory. What is required for the indispensability argument to succeed is that mathematics be explanatory in a particular way. I will argue that if heavy duty platonism is true, then the explanatory role of mathematics entails the existence of mathematical objects. On the other hand, according to the preconceived view the explanatory role of mathematics does not support a commitment to the existence of mathematical objects. Section 7 points out that the adoption of the preconceived view might help to vindicate another thesis that attracts many critics of the indispensability argument: the idea that mathematical theories are conservative over nominalistic ones. Section 8 concludes by noticing that supporters of IA are committed to many theses that are in harmony with a certain metaphysical picture (HDP), whereas critics of IA are committed to many theses that are in harmony with an opposing metaphysical view (the preconceived view). This supports the conjecture that the debate over IA is driven by the adoption of rival metaphysical views about the nature of mathematical objects by the two parties in the debate. In turn, this suggests that in order to make progress in the debate we should look for arguments which might decide whether one of these two rival metaphysical doctrines is superior to the other. 


\section{Preconceived objects}

The expression "preconceived objects" comes from Yablo 2010 (Introduction). An object o is preconceived, according to Yablo, iff "either o should have feature F, given its job description, or it does not" $(2010,7)$. This means that the features of preconceived objects are determined by the way they are characterized, where the way we characterize an object is what Yablo calls its "job description".

Looking at what Yablo considers clear examples of objects that are not preconceived can illuminate the notion of preconceived object. Consider a concrete individual like Napoleon. Napoleon has many properties which are not consequences of how we think about Napoleon: whatever the concept of Napoleon might be, Napoleon's blood type is not a consequence of our concept of Napoleon. Napoleon "surpasses expectations" (Yablo 2010, 6). Following Russell (1919, 169-170), we might compare Napoleon and Hamlet. Hamlet, if he existed, would surpass our expectations: he would have a blood type and other features which are not consequences of our concept of Hamlet; the fact that Hamlet does not surpass our expectations might be taken as an indication that Hamlet does not exist.

Napoleon surpasses our expectations, hence he exists. Hamlet would have surpassed our expectations, had he existed; he does not surpass our expectations, hence he does not exist. Both are considered by Yablo non-preconceived objects. So the existence of a nonpreconceived makes a difference in terms of the features possessed by that object. Preconceived objects are different: whether they have a certain feature depends only on the way they are characterized, not on whether they exist.

Until now I used the expression "job description" without providing a precise definition. I will say more about that in what follows, but first let me make a few points that will be important for my discussion. Yablo distinguishes between pure abstracta like the number zero and impure abstracta like \{Socrates\}. He maintains that the pure abstracta are preconceived objects, whereas $\{$ Socrates\} is only a relatively preconceived object, because its features are determined by its job description modulo the properties of Socrates.

It should be noted that even numbers are, in some sense, only relatively preconceived objects. If we consider only the relations that the number 0 entertains with other numbers, then such relations are probably determined by the job description of the number zero, i.e. something like: being the first element of the omega-sequence structure. But the job description of the number zero should probably contain also the clause: zero is the number of the Fs iff there are no Fs. So, whether zero is the number of the dragons depends on the job description of zero and on whether there are dragons. In general, the features of preconceived objects are determined by their job description and the features of non-preconceived objects. ${ }^{1}$ I am going to say more on this topic below.

Another way to distinguish preconceived and non-preconceived objects is in terms of distribution of truth-values. The line that distinguishes correct arithmetical claims and incorrect arithmetical claims is equally clear for those who believe in the existence of abstract entities as for those who do not (Yablo 2012, 1025). "There are infinitely prime numbers"

1 It might be interesting to note that there can be relatively preconceived objects that are not (impure) abstract objects. Yablo (2010, Introduction) cites mereological sums as an example: the sum of Obama's eyes and your ears is preconceived relatively to your ears and Obama's eyes. 
counts as a correct arithmetical claim, its negation as an incorrect one. This is because the job description of the natural number system entails that prime numbers must be infinitely many, so our characterization of the natural number system provides enough information to classify the sentence "There are infinitely prime numbers" as a correct one. On the contrary, the job description of the Italian prime minister is not enough to settle the truth values of claims like "the Italian prime minister is young": whether claims of this sort are true depends on contingent features of the Italian prime minister. An existing Italian prime minister "is an original source of information of the type that decides truth values" (Yablo 2010,7).

With preconceived objects the situation is different: the distribution of truth values among arithmetical sentences does not depend on the existence of numbers, in line with the idea, defended by Putnam and others, that there can be mathematical objectivity without mathematical objects (Putnam 2009, lecture 3).

The job description of the preconceived objects might be an axiomatic theory: in the case of natural numbers, second order Peano arithmetic plus some bridge principle (like that the number of the Fs $=\mathrm{N}$ iff there are $\mathrm{n} F$ ), or the theory having Hume's principle as the only non-logical axiom (Hale and Wright 2003). Or it might be that our full conception of numbers and sets is just an informal one, not captured by any axiomatic theory. Or it could be that the job description of mathematical objects is constituted by a set of postulates, conceived as procedures that allow introducing such objects into the domain of discourse, in the style of Kit Fine's procedural postulationism (Fine 2005). Supporters of the preconceived view need not take a position on what really is the job description of mathematical objects like numbers and sets. Even platonists, in order to make intelligible the claim that numbers and sets exist, need to characterize sets and numbers somehow, be it a formal or informal characterization. The same holds for anti-platonists. Supporters of the preconceived view can simply borrow the job description of the relevant mathematical objects from the other parties in the debate.

It is important to note that the preconceived view is compatible with a variety of positions. An example of a platonist position that is compatible with the preconceived view is the plenitudous platonism defended by Balaguer $(1995 ; 1998)$. On the other hand, NeoMeinongians like Graham Priest come very close to adopting the preconceived view (see Priest 2005, Ch.7). Hilbert's idea that the axioms of a formalized theory work as implicit definitions of the objects the theory is about certainly resembles the preconceived view. Finally, Zalta's idea that mathematical objects encode some properties via a mathematical theory that characterizes those objects (see Bueno and Zalta 2005) is in line with the preconceived view (even though the preconceived view per se makes no use of Zalta's distinction between encoding and instantiating a property).

A final point that should be mentioned is the connection between the preconceived view and the so-called 'open-texture' of our mathematical concepts (Waissman 1965). If the preconceived view is correct, the features of mathematical objects like numbers and sets are fixed by our conceptions of numbers and sets. But our conceptions of numbers and sets can vary and we might discover that what we currently take as the job description of numbers and sets might change due to the development of mathematical knowledge. Even though some supporters of the preconceived view are bothered by the open texture of our mathematical concepts (see Yablo 2010, 8-9), it should be noted that there is no contradiction between the open texture of our mathematical concepts and the preconceived view: 
the preconceived view holds that the features of numbers and sets are fixed by their job description, not that the job description of numbers and sets is fixed once and for all.

\section{Heavy duty platonism ${ }^{2}$}

Heavy duty platonism (HDP) is a view about the metaphysics of physical magnitudes, like temperature and mass. The most recent discussion of HDP is Knowles (2015), which characterizes HDP as "the view that physical magnitudes, such as mass and temperature, are cases of physical objects being related to numbers" (Knowles 2015, 1255).

It is important to distinguish HDP from weaker versions of platonism. All platonists believe in platonic relations, i.e. "relations of physical magnitude that relate physical things and numbers" (Field 1989, 186). The difference between HDP and weaker versions of platonism has to do with:

what they tell us about these relations: weaker forms tell us they are derivative of more fundamental properties or relations that hold of physical objects alone, while HDP says these relations are fundamental. (Knowles 2015 1256)

According to weaker versions of platonism this chair bears the mass-in- $k g$ relation to the number 8 because this chair has-mass-8-Kg (mind the hyphens!) and has-mass-8-kg is a nominalistic property: a property that holds 'of the physical object alone' as Knowles says. The obtaining of platonic relations between concreta and abstracta, on standard versions of platonism, is grounded in the nominalistic properties possessed by concreta (see Liggins 2016).

HDP differs from weak versions of platonism because according to HDP platonic relations between abstracta and concreta are metaphysically fundamental, they do not hold in virtue of any nominalistic properties possessed by the concrete objects alone.

The preconceived view and HDP are in contrast with each other. The preconceived view holds that the relations between abstract mathematical objects and concrete objects depend on the job description of the mathematical objects and the features of the concrete objects. According to HDP, on the other hand, physical magnitudes are constituted by the relations between concrete objects and numbers and such relations "do not supervene on the totality of facts either about the concrete realm or about the narrowly mathematical relations with which M [the relevant mathematical theory] is concerned" (Dorr 2010, 148). In other words, according to HDP, numbers are not (relatively) preconceived objects. Let me elaborate on this point.

\section{The contrast between the two views}

In recent work on metaphysics, the term "grounding" refers to a relation of non-causal dependence, or metaphysical dependence, usually introduced by the use of terms like "in vir-

2 This section overlaps with Plebani (2017, section 4). 
tue of" or "because" when not used in a causal sense (see Clark and Liggins (2012) for an introduction to grounding and Liggins (2016) for a discussion of the connections between grounding and IA). I am going to assume that the relata of the grounding relations are facts to simplify the exposition, but nothing important hinges on that. What matters is that recent work on grounding should make the following thesis intelligible:

- (Grounding) The possession of physical properties by concrete objects is not grounded in the obtaining of relations between concrete and abstract objects (platonic relations). Rather, platonic relations are grounded in the nominalistic properties of concrete objects.

(Grounding) is the view concrete objects do not have their physical properties in virtue of facts that entail the existence of mathematical objects (it is the other way round), where the meaning of "in virtue of" is the one that grounding theorists give to that expression.

HDP is in tension with (Grounding): HDP is precisely the view that some relations between numbers and concrete things are not "derivative of more fundamental properties or relations that hold of physical objects alone" (Knowles 2015, 1256). In the rest of this section I will argue that friends of the preconceived view should be attracted to (Grounding).

The reason why friends of the preconceived view should be attracted to (Grounding) is that, according to the preconceived view, mathematical objects are relatively preconceived objects, which means that the features of a mathematical object (its properties and its relations to other objects) are the result of two factors: (i) the job description of the mathematical object; (ii) the features of some un-preconceived objects. For example, modulo the job description of sets, the features of $\{\{$ Socrates $\}\}$ are determined by the features of $\{$ Socrates $\}$, which are determined by the features of Socrates. According to the preconceived view, the features of an impure set are determined by its job description and the features of the elements of the set; as long as set-membership is a well-founded relation and grounding is a transitive one, the features of impure sets are ultimately grounded in the features of non-sets (plus the job description of sets). If relations between concrete and abstract objects are conceived as impure sets, then the preconceived view holds that the relations between abstract and concrete objects are grounded in features of the concrete objects alone. As long as grounding is an anti-symmetric relation, the fact that relations between the abstract and the concrete are grounded in facts involving only concrete objects entails that the physical properties of concrete objects are not grounded in the relations they bear to mathematical objects.

It is also worth noticing that (Grounding) supports the following thesis:

- (Concrete-contingency) The existence of mathematical objects is neither concretely-necessary nor concretely-impossible.

(Concrete-contingency) employs a modality distinct from metaphysical possibility, i.e. concrete possibility (Yablo 2012, Dorr 2010). Something is concretely possible if it is not ruled out by facts having to do with how the concrete world is. That something is concretely possible does not mean that it is metaphysically possible: even if C, the totality of truths about the concrete world, does not entail $\neg p$, perhaps there is a metaphysical truth $\mathrm{M}$ such that $\mathrm{C}+\mathrm{M}$ entail $\neg \mathrm{p}$. The distinction between concrete and metaphysical possibility is modelled on Fine's distinction between essential and merely necessary properties: according to Fine, it is metaphysically necessary that Socrates exist if and only if $\{$ Socrates\} 
exists, but the existence of $\{$ Socrates $\}$ is not a consequence of the essence of Socrates (see Fine 1994, Dorr 2010).

Here is how Grounding supports Concrete-contingency. The preconceived view takes the relation between Socrates and \{Socrates\} as the model of the relations between concrete and mathematical objects in general. Concrete-contingency and Grounding are naturally combined because they are two ways to describe an asymmetry in the relation between, e.g., Socrates and its singleton: the singleton of Socrates depends on Socrates in a way in which Socrates does not depend on its singleton. If it were part of the essence of Socrates to be member of his singleton, then Socrates would be ontologically dependent on its singleton; but according to Grounding, Socrates does not depend on its singleton, it is the other way round. ${ }^{3}$ The preconceived view is committed to this kind asymmetric dependence of the abstract world on the concrete world, which motivates at the same time Grounding and Concrete contingency.

\section{The indispensability argument and the nature of mathematical objects}

The indispensability argument (IA) can be presented as an argument for the existence of abstract mathematical objects (see Liggins 2016, fn. 2 for discussion). It is also natural to take as one of the premises of IA the observation that mathematics is indispensable to science in some sense. This suggests that the crudest formulation of the argument should be something along these lines (see Liggins 2016, 532):

(1) Mathematics is indispensable to science [in some sense].

(2) If mathematics is indispensable to science [in the sense of 'indispensable' used in premise 1], then there are mathematical objects.

Therefore: there are mathematical objects.

Here is the connection between the metaphysical views discussed in sections 1 and 2 and the indispensability argument. If we adopt the preconceived view, (1) is plausible, almost trivial, but (2) is not. We might need to formulate our theory mathematically; but if mathematical objects are preconceived the truth-values of our mathematical sentences do not depend on the existence of mathematical objects.

On the other hand, HDP is in harmony with both premise (1) and premise (2). Mathematics is conceived by HDP as a science describing some fundamental relations between abstracta and concreta. If HDP is correct, then there is a sense in which mathematics is indispensable for our best scientific theories (as maintained by premise (1)), given that our best scientific theories should account for the fundamental metaphysical relations that hold between abstracta and concreta. The way in which mathematics is indispensable to science, according to HDP, is by tracking fundamental metaphysical relations between concrete objects and mathematical ones and mathematics can play this role only if there are

3 Correia 2006 (fn.6) draws a similar connection between ontological dependence and what lies in the essence of a thing: "I understand ontological dependence as follows: $x$ ontologically depends upon y iff $\mathrm{x}$ is by its very nature related to $\mathrm{y}$, i.e., iff there is a relation $\mathrm{R}$ such that it is part of the nature of $\mathrm{x}$ that Rxy." (Correia 2006 fn.6, 766) 
fundamental relations between concreta and mathematicalia. This means that according to HDP the indispensability of mathematics really entails the existence of mathematical entities, as maintained by premise (2).

I am not suggesting that friends of the indispensability argument should use HDP to defend the premises of IA. Rather, I am arguing that the premises of IA are in harmony with a certain metaphysical picture and in tension with another. Clarifying which of the two metaphysical views is the most attractive might help to evaluate the tenability of the premises of IA. In any case, for the moment what I am really interested in is the connection between IA and the two rival metaphysical views.

The conjecture that there is a link between the indispensability argument and HDP receives further support by looking at some issues connected with IA. In the following sections, I will present some theses friends of the indispensability argument subscribe to and show that such theses are easily motivated by adopting HDP and hard to combine with the preconceived view. This suggests that friends of IA have reasons to adopt HDP.

\section{Orthogonality}

There is a certain line of criticism of the indispensability argument that is inspired by the idea that the existence of mathematical objects 'makes no difference' for the way the concrete world is. Baker (2003) is a powerful critique of some ways in which 'make no difference' claims have been defended.

I agree with Baker (2003) that there is a tight connection between the indispensability argument and 'make no difference' claims, and in particular I agree with him that friends of IA are committed to the denial of MND while some prominent critics of IA are committed to MND. But I think that we should understand MND claims in a way that is different from the one proposed by Baker. ${ }^{4}$

One reason why I prefer my formulation of MND is that it might be closer in spirit to what proponents of MND have in mind (Yablo (2012) seems to confirm this impression). Or at least, they might consider adopting my formulation, if it turned out that it is immune to Baker's objection to his own formulation of MND. In any case, the following formulation of MND is clearly relevant to the debate over IA, so it is worth considering it in any case. Here is how I propose to understand MND claims:

- (Orthogonality) The two subject matters how the concrete world is and whether there are abstract objects are orthogonal. ${ }^{5}$

4 David Liggins makes a similar point: "Baker interprets the sort of difference-making at issue here as counterfactual dependence: 'if there were no mathematical objects, then the physical world would be just the same'. [...] But perhaps the difference-making in question is better understood as metaphysical dependence-the idea that things do not have their physical properties in virtue of anything to do with mathematical objects." (Liggins 2016, 534-535). Liggins' formulation of MND in terms of the notion of metaphysical dependence is close to the formulation of MND adopted here. See below. For criticism of Baker's formulation of MND, see also Yablo 2017.

5 I will use boldface for names of subject matters. 
In order to make orthogonality intelligible, definitions of the notion of subject matter and the orthogonality relation are called for. For the purposes of this paper, it might be easier to stick with Lewis' original definition of subject matter (Lewis 1988, see also Yablo 2012).

Lewis defines a subject matter as an equivalence relation between worlds: two worlds are m-equivalent if they agree on how matters stand with respect to the subject matter $\mathbf{m}$. An equivalence relation induces a partition of the logical space, i.e. a set of cells such that each world belongs to one and only one cell. Each cell is a set of worlds, so (thinking of propositions as sets of worlds) the partition associated with the subject matter $\mathbf{m}$ can be thought of as a set of propositions; we can think of these propositions as answers to the question how are matters $m$-wise?, so the subject matter $\mathbf{m}$ turns out to be a collection of answers, what is sometimes called a question (Yablo 2014, 28). The subject matter the number of stars is just the set of all the possible answers to the question how many stars are there? to take Lewis' own example. Two worlds are $\mathbf{m}$-equivalent if the answer to the question associated with $\mathbf{m}$ is the same in the two worlds. Finally, two subject matters $\mathbf{m}$ and $\mathbf{n}$ are orthogonal if every $\mathbf{m}$-cell intersects every $\mathbf{n}$-cell, which "means at an intuitive level [...] that how matters stand $\mathrm{m}$-wise puts no constraints on how they stand n-wise, or vice versa” (Yablo 2014, 29).

So orthogonality is the view that the way the concrete world is does not demand nor preclude the existence of abstract mathematical objects (Yablo 2012): given a world w in which mathematical objects are present (absent) there is a world w' in which mathematical objects are absent (present) and that is concretely indiscernible from w.

Anti-platonists have been attracted by orthogonality, but, as Baker (2003) points out, the usual argument for orthogonality is unsatisfactory. The kind of argument Baker criticizes can be schematically presented like this:

- (BAD-ProO) Mathematical entities have no causal powers; therefore the existence of mathematical entities makes no difference for how the concrete world is.

(BAD-ProO) infers that mathematical objects make no difference for the concrete world from the fact that mathematical objects make no difference for the concrete world at the causal level. But causal dependence is not the only kind of dependence: there might be some notion of dependence such that the way the world is in concrete respects depends on the existence of abstract objects.

Also the usual argument against orthogonality can be criticized. We live in a complex world and it is plausible to argue that in order to represent such a complex world we need to use terms that aim to refer to numbers, functions, sets... The problem for the deniers of orthogonality is how to spell out the idea that the actual world is so complex that it needs to include mathematical objects. Yablo (2012) points out that the following argument against orthogonality does not work:

- (BAD-AntiO) "we cannot imagine-without-numbers a complex world" therefore "we cannot imagine a complex world lacking in numbers" (Yablo 2012, 1014)

BAD-AntiO confuses the issue of what it takes to describe certain circumstances with the issue of what it takes for those circumstances to obtain. As Yablo points out, BAD-Anti-O has a similar structure as an infamous argument from Berkeley: "we cannot imagine a tree non-perceptually" therefore "we cannot imagine unperceived trees" (Yablo 2012, 1014).

Despite its importance in the debate about the indispensability argument, cogent arguments in favor or against orthogonality are lacking. I do not think there are easy arguments 
for or against orthogonality, but I submit that the two metaphysical pictures discussed above can be used to motivate orthogonality or the rejection of orthogonality. I will first argue that HDP entails the negation of orthogonality and then I will argue that friends of the preconceived view have reasons to accept orthogonality.

That HDP is in tension with orthogonality should be fairly clear. HDP entails that some features of concrete objects, like their mass or temperature, are fundamental relations between those objects and numbers. This means that concrete objects that in the actual world have certain masses or temperatures cannot retain those features in a world without numbers: there is no numberless world that is concretely indiscernible from the actual world, if HDP is correct. But this means that HDP entails the negation of orthogonality. This should not sound surprising: many authors, including supporters of orthogonality, acknowledge that HDP is in tension with orthogonality: see Rayo $(2015,70)$, Dorr (2010, 148), Yablo (2012, fn 16). Perhaps the tension between HDP and orthogonality was not perceived by friends of (Orthogonality) as a deep problem, given that HDP is usually taken to be untenable. But, as Knowles (2015) convincingly argues, the usual reasons offered to reject HDP are far from conclusive. As far as HDP is a serious option, deniers of orthogonality should consider adopting HDP.

Adopting the preconceived conception provides reasons to accept orthogonality. The reason is that the preconceived view is naturally combined with (Grounding) and (Grounding) supports (Concrete-contingency). But (Concrete-contingency) strongly supports (Orthogonality). If mathematical objects are concretely-contingent, then given a concretely-possible world $w$ in which mathematical objects are present (absent) there is a concretely-possible world w' in which mathematical objects are absent (present) and that is concretely indiscernible from $\mathrm{w}$. (Concrete-contingency) is the version of (Orthogonality) in which "possible world" is replaced by "concretely- possible world".

Summing up: friends of IA should reject (Orthogonality). The rejection of (Orthogonality) is in harmony with HDP and in tension with the preconceived view. This suggests that friends and critics of IA might be attracted by different metaphysical views.

\section{Mathematical explanations}

One cherished theme in recent debates over the indispensability argument is the idea that mathematics plays an "indispensable explanatory role" in our best scientific theories (see Baker 2009; Lyon 2012; Psillos 2010, 2011 among others) and that anti-platonists cannot account for this contribution of mathematics to our best theories. In this section I will use the previous discussion of the preconceived view and HDP to support a point that others have already made: "indispensable explanatory role' can mean different things, only some of which support realist commitment" (Saatsi 2017, 893).

The first thing to notice is that the preconceived view is not incompatible with the idea that there are mathematical explanations of empirical phenomena. Even those friends of the preconceived view who are anti-platonists do not reject mathematics; they reject mathematical objects (Azzouni 2012, 964; Yablo 2012, 1023). If some theorem of number theory or graph theory helps to explain some empirical phenomenon, this is not a problem for the anti-platonist as long as there are anti- platonist accounts of number theory and graph theory, and such accounts exist (Hellman 1989, Correia 2006, Yablo 2002). Such anti-pla- 
tonist accounts of mathematics are in line with the idea, typical of the preconceived view, that the distinction between true and false mathematical sentences does not require a commitment to the existence of mathematical objects. ${ }^{6}$

That one can accept a mathematical theory without accepting mathematical objects seems particularly plausible when one is dealing with algebraic theories, like group theory or Boolean algebra (Yablo 2012). Perhaps the thought that one cannot accept a theory without accepting its ontological commitments has some plausibility when we are dealing with theories that have an intended model, like arithmetic. But algebraic theories have no intended model, so it is not clear which are the ontological commitments of those theories. Suppose that some results from Boolean algebra help to explain some empirical phenomenon; does this prove that we should believe the ontological commitments of Boolean algebra? Perhaps, but which are exactly the ontological commitments of Boolean algebra? Do they include the commitment to the existence of abstract objects?

According to the preconceived view, there is a gap between the explanatory role of mathematics and the existence of mathematical objects. The situation changes if we turn our attention to HDP. Suppose HDP is correct and physical magnitudes are fundamental relations between abstract and concrete objects. It seems plausible that reference to physical magnitudes is indispensable for our best scientific explanations; but on the assumption that HDP is true this means that reference to abstract objects is indispensable for our best scientific explanations and hence that there are abstract mathematical objects which our theories refer to.

Similarly, if some high-order properties of concrete objects are constituted by the relations that these concrete objects bear to numbers and if explanations that make reference only to these very general properties of the concrete objects are superior to explanations that cite irrelevant details (Pincock 2007), then we have an argument for platonism. But the argument relies on the idea that some high-order properties of concrete objects are constituted by some relations holding between concrete and abstract objects: a thesis that seems very close to HDP.

The issue of mathematical explanation thus seems another case where the position of friends of the indispensability argument is in harmony with HDP and in tension with the preconceived view.

\section{Conservativeness}

Orthogonality and concrete-contingency can be split into two components: the idea that the physical world does not demand the existence of mathematical objects and the idea that the physical world does not preclude the existence of mathematical objects.

The idea that the conditions of the physical world do not preclude the existence of mathematical objects is strictly connected with the idea, defended by Field (1980, 2016)

6 One could argue that the aforementioned accounts of number-theory or graph theory are unsatisfactory, but if anti-platonists lack a satisfactory account of number theory that is a problem independently of whether number theory plays an explanatory role or not. 
that good mathematical theories should be conservative with respect to nominalistic theories (theories which only quantify over/ refer to concrete objects). One way to define conservativeness is to say that a mathematical theory $\mathrm{M}$ is conservative if and only if it is consistent with all nominalistic consistent theories. ${ }^{7}$ If we shift our attention from theories to possible worlds, we can formulate conservativeness by saying that each way the concrete world could be is compatible with the existence of mathematical objects, i.e. each cell of subject matter the concrete world overlaps with the sets of worlds in which there are mathematical objects - and this kind of overlap is indeed a consequence of orthogonality (see Yablo 2012, fn. 14).

Melia (2006) complains that Field has not provided persuasive arguments to hold that mathematics is conservative over nominalistic theories. In this section I will argue that friends of the preconceived view might have at least a strong philosophical motivation to hold that mathematics is conservative over nominalistic theories.

Let me try to make the discussion concrete by considering one worry you might have about conservativeness and how friends of the preconceived view might reply to it. Here is the worry:

If, as defenders of the conservativeness claim believe, it is right that mathematics should not have any implications about the concrete world then, in particular, mathematics should not imply any limitation on how many concrete individuals there are. [...] But the assumption that the urelements form a set does place a limit upon the size of the concrete domain. (Melia 2006, 206)

I want to argue that:

(i) There is a conception of sets that rejects the idea that certain objects might be too many to form a set. On such a conception of sets, the fact that urelements form a set does not place any limitation upon the size of the concrete domain;

(ii) The conception of sets that rejects the limitation of size principle is more fruitful, from the point of view of the preconceived view, than the conception of sets that accepts the limitation of size view;

Therefore:

(iii) Friends of the preconceived view should adopt a conception of sets according to which the existence of a set of urelements does not pose any limitation upon the size of the concrete realm.

Concerning (i). A conception of sets that rejects the idea that there could be too many objects to form a set has been defended by a variety of authors: see Linnebo $(2010, \mathrm{fn} .5)$ for a list (see also Linnebo 2010 for an attack to the limitation of size view). ${ }^{8}$ According to such a conception of sets "given any objects $\mathrm{xx}$, it is possible to form a set whose elements are precisely these objects" (Linnebo 2010,157).9

7 See Field (2016, sec. 0.4) and Melia (2006) for a more precise definition of conservativeness and some important details.

8 See also Linnebo (2013) on the "potential hierarchy of sets".

9 I am using "xx" as a plural variable (see Linnebo 2010, 2013). 
Concerning (ii): The idea that there cannot be a set of the $\mathrm{xx}$, despite the fact that it is perfectly determined which objects are the $\mathrm{xx}$, has been rejected as conflicting with "something too deeply rooted in our use of set-like operations" (Hellman 1989, 74).

If the true nature of sets was a consequence of hidden metaphysical truths, perhaps one could accept the limitation of size view: the fact that some objects are too many to form a set would just be a brute metaphysical fact. But as long as the nature of sets is a consequence of how we conceive them, as the preconceived view maintains, we should prefer the conception of sets that strikes us as more natural.

This means friends of the preconceived view should not worry about possible cases of failure of conservativeness like those mentioned in the quote from Melia. I think that the point generalizes: friends of the preconceived view should find the claim that mathematics is conservative congenial to their views on the nature of mathematical objects. Here is why.

According to the preconceived view, the features of a mathematical object are determined by its job description. I think that friends of the preconceived view should also be attracted by the following thesis:

- (Cons) We should look for job descriptions of mathematical objects that make the existence of an object satisfying that description compatible with any way the concrete world might be.

The preconceived view holds that the properties of impure mathematical objects depend on how the concrete objects are. This removes one possible way in which the existence of mathematical objects might conflict with how the concrete world is: impure mathematical objects are not characterized independently from how the concrete world is; rather they adapt themselves to the way the concrete world is. According to the preconceived view, the job description of an impure mathematical object specifies the way that impure mathematical object is as a function of how non-mathematical objects are.

Of course, this falls short of a proof that our actual mathematical theories are conservative or even that the best mathematical theories are always conservative. But what I said should be enough to justify the claim that according to the preconceived view the conservativeness of our mathematical theories counts as a desirable goal. And the preceding discussion of the limitation of size view should at least give us some hope that mathematical objects can be characterized in a way that makes their existence compatible with every possible concrete situation.

\section{Conclusions}

I have sketched two pictures of the nature of mathematical objects: HDP and the preconceived view. I have argued that HDP supports many theses that friends of the indispensability argument are committed to, while the preconceived view is in tension with the commitments of friends of the indispensability argument. This supports the conjecture put forward in the introduction: that friends and critics of IA are attracted by different metaphysical pictures. One moral of the paper is that it might be worth connecting the discussion of the indispensability argument with the discussion of HDP (and rival positions). Joseph Melia reached a similar conclusion: 
Of course I (like Field) find the Heavy Duty Platonism countenanced here implausible. But if you could convince me of it, I would retract my view [...] However, it's precisely these kind of metaphysical debate which philosophers should be focusing upon when trying to discover what there is..(Melia 1995, 229)

We saw that connecting the discussion of IA with the discussion of HDP also illuminates many topics connected with IA: orthogonality, grounding, explanation and conservativeness. This further supports Melia's insight and the idea that the contrast between HDP and rival metaphysical views is of "great dialectical importance" (Knowles 2015, 1255).

\section{REFERENCES}

Azzouni, Jody. 2012. Taking the easy road out of dodge. Mind 121/484: 951-965.

Baker, Alan. 2003. Does the existence of mathematical objects make a difference? Australasian Journal of Philosophy 81/2: 246-264.

-. 2009. Mathematical explanation in science. The British Journal for the Philosophy of Science 60 (3): 611-633.

Balaguer, Mark. 1995. A platonist epistemology. Synthese 103/3: 303-325.

-. 1998. Platonism and anti-Platonism in mathematics. Oxford: Oxford University Press.

Bangu, Sorin. 2017. Indispensability of mathematics. OBO. Retrieved 29 Sep. 2017, from http://www. oxfordbibliographies.com/view/document/obo-9780195396577/obo-9780195396577-0241.xml.

Bueno, Otávio and Edward N. Zalta. 2005. A nominalist's dilemma and its solution. Philosophia Mathematica 13/3: 297-307.

Clark, Michael J. and David Liggins. 2012. Recent work on grounding. Analysis 72: 812-823.

Colyvan, Mark. 2015. Indispensability arguments in the philosophy of mathematics. In Edward N. Zalta, ed., The Stanford Encyclopedia of Philosophy (Spring 2015 Edition) https://plato.stanford.edu/archives/ spr2015/entries/mathphil-indis/.

Correia, Fabrice. 2006. Generic essence, objectual essence, and modality. Nous 40/4: 753-67.

Dorr, Cian. 2010. Of numbers and electrons. Proceedings of the Aristotelian Society 110/2pt2: 133-181.

Field, Hartry. 1989. Realism, mathematics and modality. Oxford: Blackwell.

-. 2016 (2nd ed.). Science without numbers. Oxford: Oxford University Press.

Fine, Kit. 1994. Essence and modality. Nous Supplement 8: 1-16.

-. 2005. Our knowledge of mathematical objects. In Tamar Szabo Gendler and John Hawthorne, eds., $O x$ ford studies in epistemology, 89-109. Oxford: Clarendon Press.

Hale, Bob and Crispin Wright. 2003. Reason's proper study: Essays towards a neo-Fregean philosophy of mathematics. Oxford: Oxford University Press.

Hellman, Geoffrey. 1989. Mathematics without numbers: Towards a modal-structural interpretation. Oxford: Oxford University Press.

Knowles, Robert. 2015. Heavy duty platonism. Erkenntnis 80/6: 1255-1270.

Lewis, David. 1988. Statements partly about observation. Philosophical Papers 17/1: 1-31.

Liggins, David. 2016. Grounding and the indispensability argument. Synthese 193/2: 531-548.

Linnebo, Øystein. 2010. Pluralities and sets. Journal of Philosophy 107/3: 144-164.

-. 2013. The potential hierarchy of sets. Review of Symbolic Logic 6/2: 205-228.

Lyon, Aidan. 2012. Mathematical explanations of empirical facts, and mathematical realism. Australasian Journal of Philosophy 90/3: 559-578.

Melia, Joseph. 1995. On what there's not. Analysis 55/4: 223-229.

-. 2006. The conservativeness of mathematics. Analysis 66/3: 202-208.

Pincock, Chris. 2007. A role for mathematics in the physical sciences. Noûs 41/2: 253-275. 
Plebani, Matteo. 2017. Mathematical platonism meets ontological pluralism? Inquiry DOI: 10.1080/ 0020174X.2017.1347518

Priest, Graham. 2005. Towards non-being: The logic and metaphysics of intentionality. Oxford: Oxford University Press.

Psillos, Stathis. 2010. Scientific realism: Between platonism and nominalism. Philosophy of Science 77/5: 947958.

-. 2011. Living with the abstract: realism and models. Synthese 180/1: 3-17.

Putnam, Hilary. 2009. Ethics without ontology. Cambridge: Harvard University Press.

Rayo, Agustín. 2015. Nominalism, trivialism, logicism. Philosophia Mathematica 23/1: 65-86.

Russell, Bertrand. 1919. Introduction to mathematical philosophy. London: George Allen and Unwin.

Saatsi, Juha. 2017. Dynamical systems theory and explanatory indispensability. Philosophy of Science 84/5: 892-904.

Yablo, Stephen. 2002. Abstract objects: A case study. Nous 36/s1: 220-240.

-. 2010. Things: Papers on objects, events, and properties. Oxford: Oxford University Press.

-. 2012. Explanation, extrapolation, and existence. Mind 121/484: 1007-1029.

-. 2014. Aboutness. Princeton: Princeton University Press.

-. 2017. If-Thenism. Australasian Philosophical Review 1 (2): 115-132. DOI: 10.1080/24740500.2017.1346423

Waismann, Friedrich. 1965. Verifiability. In Antony Flew, ed., Logic and language, 122-51. New York: Doubleday.

Matteo Plebani works on topics at the intersection between the philosophy of mathematics, metaphysics and the philosophy of language, like the platonism-nominalism debate in the philosophy of mathematics and recent work on the notions of subject matter and partial truth.

Adress: Department of Philosophy, University of Amsterdam, Oude Turfmarkt 145-147, 1012 GC Amsterdam, Netherlands. Email: plebani.matteo@gmail.com 